\title{
Interdigitated craniotomy: a simple technique to fix a bone flap with only a single plate
}

\author{
Noboru Takahashi, MD, PhD, ${ }^{1}$ Kazunori Fujiwara, MD, PhD, ${ }^{1}$ Keiichi Saito, MD, PhD, ${ }^{1}$ and \\ Teiji Tominaga, MD, PhD²
}

\begin{abstract}
'Department of Neurosurgery, Iwate Prefectural Iwai Hospital, Ichinoseki; and 2Department of Neurosurgery, Tohoku University Graduate School of Medicine, Sendai, Japan
\end{abstract}

In pterional craniotomy, fixation plates cause artifacts on postoperative radiological images; furthermore, they often disfigure the scalp in hairless areas. The authors describe a simple technique to fix a cranial bone flap with only a single plate underneath the temporalis muscle in an area with hair, rather than using a plate in a hairless area. The key to this technique is to cut the anterior site of the bone flap at alternate angles on the cut surface. Interdigitation between the bone flap and skull enables single-plate fixation in the area with hair, which reduces artifacts on postoperative radiological images and provides excellent postoperative cosmetic results.

http://thejns.org/doi/abs/10.3171/2014.10.JNS141034

KEY WORDS craniotomy; fixation; plate; surgical technique

A $\mathrm{N}$ objectionable bulge is a common sequela of cranial fixation plates, especially in patients with a thin scalp. To prevent this undesirable deformity in pterional craniotomy, we developed interdigitated craniotomy as an alternative to plate fixation at the anterior site of a cranial bone flap.

\section{Operative Technique}

The skin and temporalis muscle are incised in the standard fashion for pterional craniotomy and then reflected inferiorly as a single layer. With a 4-mm cutting bur, the initial cut is made linearly from the lateral greater wing of the sphenoid to the inferior part of the temporal bone. A MacCarty keyhole ${ }^{5}$ is not made; however, a bur hole is placed in the posterior section of the temporal bone at the squamous suture, and the bone dust is collected for later use. The dura mater is then stripped from the undersurface of the bone flap. The second cut is made with a craniotome at the posterior region of the temporal bone. The final cut is made at the parietal and frontal bones. While cutting the frontal bone, the blade of the craniotome is used with an oscillating movement, resulting in alternate angles at the cut surface. Then, the free bone flap is raised (Fig. 1), and the inner surface of the sphenoid wing is removed with the cutting bur. On closure, the beveled edges of the bone flap and skull are interdigitated with a small amount of the bone dust interposed between the edges, and then the posterior site of the bone flap is fixed with a circular titanium plate and screws to cover the single bur hole (Figs. 2 and 3). Small grooves in the frontal bone and sphenoid wing are filled with the remaining bone dust.

\section{Results}

Nine patients ( 2 men and 7 women) with a mean age of 62 years (median 64 years, range 39-78 years) underwent interdigitated craniotomy between January 2014 and May 2014. Lesions included 8 anterior circulation aneurysms and 1 putaminal hemorrhage.

The quality of the bone flap fixation was assessed at least 3 months after the surgery. At the hairless area, the positioning of the bone flap with respect to the height of its surface relative to the surface of the adjacent bone (offset) was assessed by appearance and palpation.

The patient characteristics and results of the follow-up assessment are provided in Table 1. Excellent results were achieved in all patients, except for 1 patient who died dur-

SUBMITTED June 6, 2014. ACCEPTED October 20, 2014.

INCLUDE WHEN CITING Published online April 17, 2015; DOI: 10.3171/2014.10.JNS141034.

DISCLOSURE The authors report no conflict of interest concerning the materials or methods used in this study or the findings specified in this paper. 


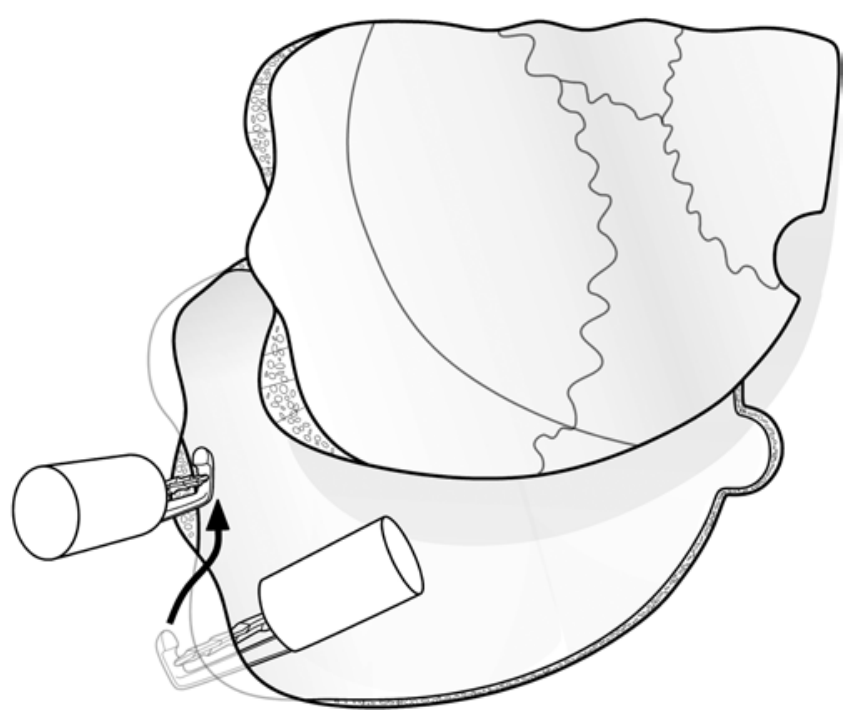

FIG. 1. Illustration of interdigitated craniotomy. Alternate angles are made at the cut surface of the frontal bone by using an oscillating movement of the craniotome blade. Copyright Noboru Takahashi. Published with permission.

ing acute subarachnoid hemorrhage. Offset was not visually apparent or felt by palpation at the hairless areas. The internal review board at the Iwate Prefectural Iwai Hospital approved the study.

\section{Discussion}

Plate fixation is a straightforward and secure procedure in the restoration of cranial bone flaps. Two or 3 plates are

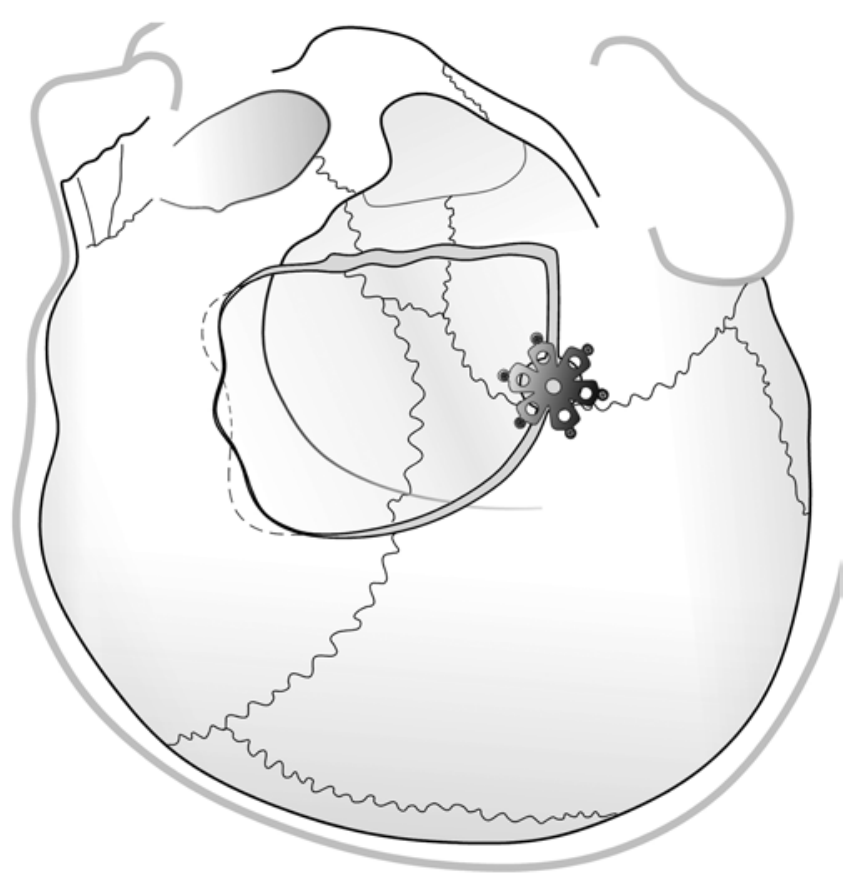

FIG. 2. Illustration showing restoration of the bone flap. The beveled edges of the bone flap and skull are interdigitated, and the posterior site of the bone flap is fixed with a circular titanium plate and screws covering the single bur hole. Copyright Noboru Takahashi. Published with permission.
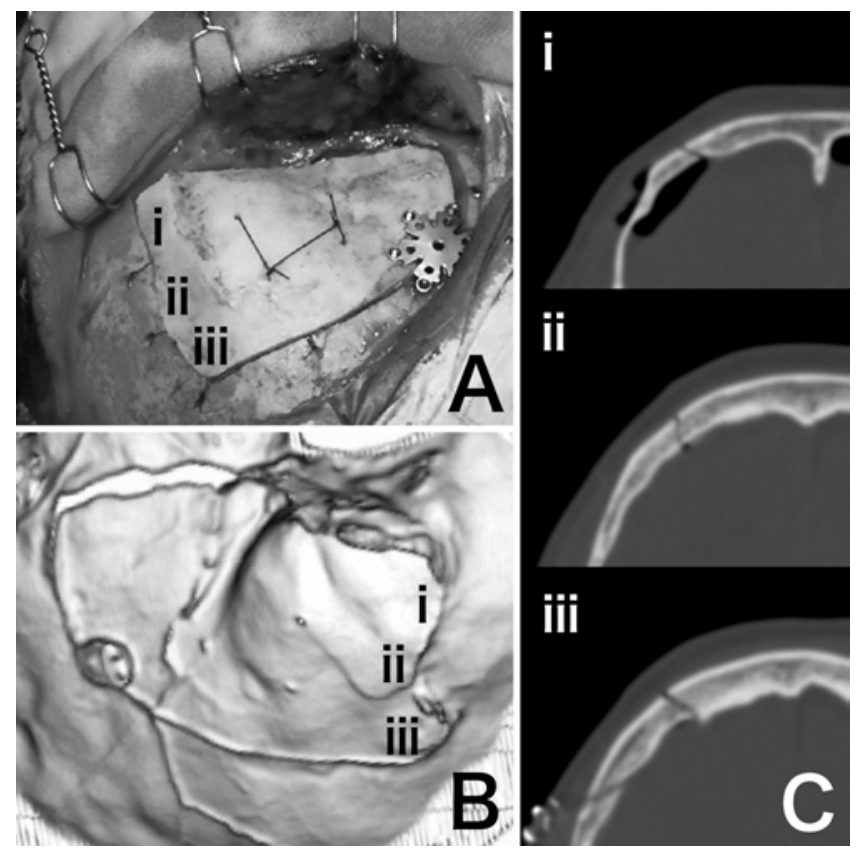

FIG. 3. Intraoperative photograph and postoperative CT scans illustrating the interdigitation between the bone flap and skull in a right pterional craniotomy. The lower (i), middle (ii), and upper (iii) edges of the cut surface of the frontal bone are interdigitated with alternate angles. A: Intraoperative photograph showing the bone flap fixed to the skull. B: Postoperative 3D CT scan of the inside view of the skull. C: Postoperative axial bone CT scans showing the angles of the cut surfaces.

typically used to fix the bone flaps firmly to the skull. ${ }^{8}$ However, when placed under the scalp in hairless areas, plates can disfigure the contour of the skin and appear as objectionable bulges, particularly in patients with a thin scalp.

The superiority of titanium plate fixation in comparison with wire fixation for cosmesis has been described by Broaddus et al. ${ }^{1}$ Of 19 patients with titanium plate fixation who were followed up for at least 3 months after surgery, 14 had no offset palpable (excellent results), 4 had palpable but invisible offset of less than $2 \mathrm{~mm}$ (acceptable results), and 1 patient had significant visible offset of more than $2 \mathrm{~mm}$ (unacceptable results). The bulge of the plate was not reported in the study, but in another study by Shimizu et al., the bulge associated with a $0.6-\mathrm{mm}$ titanium plate (Bioplate; Codman \& Shurtleff, Inc.) fixed by self-tapping screws was reported at approximately $1 \mathrm{~mm}$. $^{7}$ Furthermore, fitting the plate into a small groove dug on the bone so that the plate is flush with the bone ${ }^{2,7}$ is reportedly not applicable for a thin outer table?

Titanium plates can also cause skin issues such as irritation, ${ }^{9}$ necrosis, or fistula, ${ }^{3,6}$ potentially requiring a further procedure for hardware removal and reconstruction. Fukunaga et al. reported 2 cases in which patients suffered from a skin ulceration at the location of a scar from a previous operation in the midfrontal area and exposure of the implanted titanium plate, ${ }^{3}$ and Okuda et al. described 3 cases of skin necrosis at the frontotemporal region in patients who had undergone implantation of a large star-shaped titanium plate directly under the scalp at the frontal tuberosity. ${ }^{6}$ The latter authors suspected that 
TABLE 1. Clinical characteristics and follow-up results in 9 patients who underwent interdigitated craniotomy

\begin{tabular}{ccllcc}
\hline Case No. & Age $(\mathrm{yrs})$, Sex & \multicolumn{1}{c}{ Cause } & Appearance of Offset & Palpation of Offset & Follow-Up (mos) \\
\hline 1 & $64, \mathrm{M}$ & Ruptured ACoA aneurysm & None & None & 7 \\
\hline 2 & $64, \mathrm{~F}$ & Ruptured MCA aneurysm & None & None & 7 \\
\hline 3 & $49, \mathrm{~F}$ & Ruptured ACoA aneurysm & None & None & 6 \\
\hline 4 & $39, \mathrm{~F}$ & Unruptured ICA-PCoA aneurysm & None & None & 6 \\
\hline 5 & $61, \mathrm{M}$ & Ruptured ACoA aneurysm & None & None & 5 \\
\hline 6 & $78, \mathrm{~F}$ & Ruptured ACoA aneurysm & None & None & 4 \\
\hline 7 & $75, \mathrm{~F}$ & Ruptured ICA-PCoA aneurysm & None & None & 4 \\
\hline 8 & $72, \mathrm{~F}$ & Ruptured ACoA aneurysm & NA & NA & Dead \\
\hline 9 & $57, \mathrm{~F}$ & Putaminal hemorrhage & None & None & 3 \\
\hline
\end{tabular}

$\mathrm{ACOA}=$ anterior communicating artery; ICA = internal carotid artery; $\mathrm{MCA}=$ middle cerebral artery; $\mathrm{NA}=$ not applicable; $\mathrm{PCo}=$ posterior communicating artery.

pressure was placed on the scalp from below by a highly convex frontal tuberosity and that the titanium plate was under high levels of skin tension. As a result, the blood flow was insufficient, and scalp necrosis occurred. These cases highlight the risk of ulceration in hairless areas with the use of a titanium plate. Therefore, we believe that a titanium plate should not be used in hairless areas if possible.

Instead, bone flaps can be fixed using alternative methods (e.g., suture, wire, middle table pin, ${ }^{9}$ or bone shim ${ }^{10}$ ). However, a suture does not provide rigid fixation, ${ }^{4}$ whereas a wire is more likely than plates to cause an objectionable bulge. A middle table pin ${ }^{9}$ is not always applicable for a thin or osteoporotic skull, and the method of placing a bone shim ${ }^{10}$ is slightly complicated.

Therefore, interdigitated craniotomy is an alternative to the use of plates in hairless areas. Interdigitation between the bone flap and skull eliminates the danger of floating,
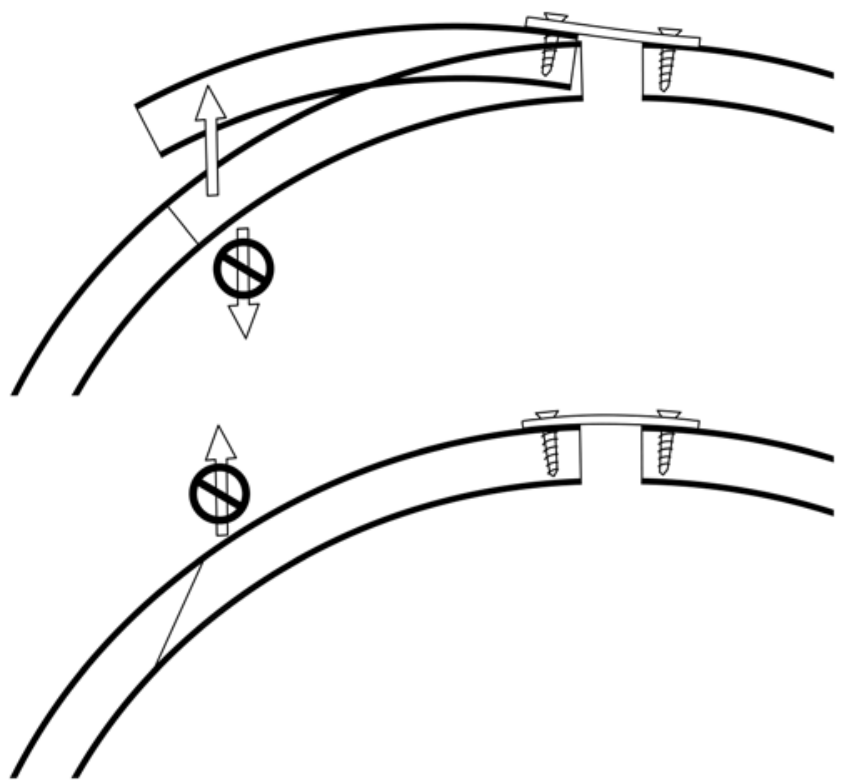

FIG. 4. Schema of a cross-section of the bone flap and skull. Upper: Depression of the bone flap can be prevented by using a perpendicular cut surface; however, the bone flap is still free to float. Lower: Floating of the bone flap is prevented with a beveled edge on the skull. Copyright Noboru Takahashi. Published with permission. depression, and horizontal displacement of the bone flap. Considering that a cranial bone flap is a spherical surface, single-plate fixation at the posterior site can prevent depression of the bone flap, even when the cut surfaces on the frontal bone are perpendicular; however, the attached edge of the frontal bone may float easily via a hingelike motion with the plate (Fig. 4 upper). The beveled edges on the skull, which are similar in appearance to eaves and are used in interdigitated craniotomy, prevent floating and horizontal displacement of the bone flap (Fig. 4 lower).

In interdigitated craniotomy, the offset is almost zero. Moreover, without a plate at the hairless area, an objectionable bulge is never generated. The bone dust interposed between the bone edges fills the slight gap and prevents slipping, and the remaining bone dust fills the grooves of the frontal bone and sphenoid wing. With this method, all of our patients had excellent results, without visible or palpable offset at the hairless areas.

Regarding the use of materials, interdigitated craniotomy uses only 1 bur hole cover plate and 4 or 5 screws, compared with an average of 1 bur hole cover plate, 2 other plates, and 8 screws for standard titanium plate fixation. ${ }^{1}$ Moreover, artificial materials to fill the bone defect such as a ceramic bur hole button, methyl methacrylate, hydroxyapatite, and calcium phosphate are not required. As a result, our method may also help to decrease operating room costs. Furthermore, the minimal use of metal reduces artifacts on postoperative radiological images.

Interdigitated craniotomy is a simple and useful technique that provides excellent cosmetic results, decreases cost, and reduces artifacts in pterional craniotomy.

\section{References}

1. Broaddus WC, Holloway KL, Winters CJ, Bullock MR, Graham RS, Mathern BE, et al: Titanium miniplates or stainless steel wire for cranial fixation: a prospective randomized comparison. J Neurosurg 96:244-247, 2002

2. Di Lorenzo N, Mouchaty H, Shamsaldin M, Gallina P, Maleci A: Cranial bone flap fixation with microplates and screws: a new application technique. Technical note. J Neurosurg Sci 48:55-56, 2004

3. Fukunaga Y, Hashimoto I, Seike T, Kashiwagi K, Nakanishi $\mathrm{H}$ : [Reconstruction of frontal deformities with calcium phosphate paste cement and temporal musculo-pericranial flap.] J Jpn Cranio-Max-Fac Surg 27:279-285, 2011 (Jpn) 
4. Lerch KD: Reliability of cranial flap fixation techniques: comparative experimental evaluation of suturing, titanium miniplates, and a new rivet-like titanium clamp (CranioFix): technical note. Neurosurgery 44:902-905, 1999

5. MacCarty CS: Meningiomas of the sphenoidal ridge. J Neurosurg 36:114-120, 1972

6. Okuda T, Akimoto M, Kuroda S, Hyakusoku H: [Three cases of skin necrosis in frontal-temporal region after neurosurgical procedure.] J Jpn Cranio-Max-Fac Surg 19:261-266, 2003 (Jpn)

7. Shimizu S, Hagiwara H, Nakayama K, Fujii K: [Technique for embedding a titanium miniplate in the forehead that avoids objectionable bulges.] No Shinkei Geka 37:43-46, 2009 (Jpn)

8. Smith SC, Pelofsky S: Adaptation of rigid fixation to cranial flap replacement. Neurosurgery 29:417-418, 1991

9. Spetzler RF: Bone flap fixation: a new technique. Technical note. J Neurosurg 87:475-476, 1997
10. Winston KR, Wang MC: Cranial bone fixation: review of the literature and description of a new procedure. J Neurosurg 99:484-488, 2003

\section{Author Contributions}

Conception and design: Takahashi. Acquisition of data: Takahashi, Fujiwara, Saito. Analysis and interpretation of data: all authors. Drafting the article: Takahashi. Critically revising the article: all authors. Reviewed submitted version of manuscript: all authors. Approved the final version of the manuscript on behalf of all authors: Takahashi. Administrative/technical/material support: Takahashi. Study supervision: Takahashi, Tominaga.

\section{Correspondence}

Noboru Takahashi, Department of Neurosurgery, Iwate Prefectural Iwai Hospital, 17 Odaira, Kozenji, Ichinoseki, Iwate 0210192, Japan. email: takahashi.iwai@gmail.com. 